\title{
Survey on the Cognition of HIVIAIDS Among Freshmen of Midwifery Major in Nursing Department
}

\author{
Liu Zhimei ${ }^{1, ~ *}$, Shen Tao ${ }^{1}$, He Shixu ${ }^{1}$, Xiao Dexin ${ }^{2}$, Zhang Xinjiang ${ }^{3,}$, Yang Ying ${ }^{3}$, Wang Zhenjuan ${ }^{3}$, \\ Sun Ying ${ }^{3}, \mathrm{Fu} \mathrm{Yu}^{3}$, Zhao Yating ${ }^{3}$, Yang Dezhi ${ }^{3}$ \\ ${ }^{1}$ Department of Cosmetology, Zunyi Medical College, Zunyi, China \\ ${ }^{2}$ Department of Dermatology, Affiliated Mou Tai Hospital of Zunyi Medical University, Zunyi, China \\ ${ }^{3}$ Department of Dermatology, Affiliated Second Hospital of Zunyi Medical University, Zunyi, China
}

Email address:

568137312@qq.com (Liu Zhimei),904107218@qq.com (Zhang Xinjiang)

${ }^{*}$ Corresponding author

\section{To cite this article:}

Liu Zhimei, Shen Tao1, He Shixu, Xiao Dexin, Zhang Xinjiang, Yang Ying, Wang Zhenjuan, Sun Ying, Fu Yu, Zhao Yating, Yang Dezhi. Survey on the Cognition of HIV/AIDS Among Freshmen of Midwifery Major in Nursing Department. American Journal of Clinical and Experimental Medicine. Vol. 9, No. 2, 2021, pp. 40-43. doi: 10.11648/j.ajcem.20210902.13

Received: March 23, 2021; Accepted: April 22 2021; Published: May 8, 2021

\begin{abstract}
Background: Midwives were a special group of nurses whose jobs were to assist obstetricians in ensuring the safety of mothers and newborns. It was important that their high knowledge of preventing human immunodeficiency virus (HIV) transmission for preventing mother-to-child transmission of HIV. In order to understand and strengthen their knowledge of HIV and acquired immunodeficiency syndrome (AIDS). When the new students of midwive first came to college the study of ther wareness for HIV was carried out. Objective: The main aim of this study was to understand the awareness rate and provide basis of health education about the AIDS among new students of midwive. Materials and Methods: 146 new students of midwives were surveyed about the knowledge of AIDS with questionnaire survey method. Result: Findings revealed that knowledge level of AIDS were low among the new students of midwives. Conclusion and Recommendation: Learning of sexual knowledge and HIV/AIDS should be strengthened for the new students of midwives. The curriculum about HIV/AIDS knowledge should be specially set up.
\end{abstract}

Keywords: HIV, AIDS, Midwife, New Student

\section{Introduction}

AIDS was a serious human immunodeficiency disease caused by infection with HIV. After human body were infected by the HIV, the immune system of the human body were destroied and resulted in the exceeding low the ability of resisting disease, which eventually leaded to systemic failure and death.

The three ways of AIDS transmission were sexual transmission, blood transmission and mother-to-child transmission. The sexual transmission was through male homosexual contact or male and female heterosexual contact. The blood transmission was through blood and blood products transfusion. Contaminated injection needles and needle tubes and surgical and dental instruments. The mother-to-child transmission was perinatal transmission through the placenta, the birth canal of childbirth and breastfeeding.

Job of the midwive was to perform normal delivery or dystocia delivery under the guidance of obstetricians every day. If the midwife had a wealth of knowledge about the pathogens, transmission routes, and hazards of HIV/AIDS and how to prevent and block the transmission of AIDS, it was of great significance to block mother-to-child transmission among the three transmission routes of AIDS.

AIDS caused by HIV infection not only brought catastrophic harm to human health, but also infected vertically through mother-to-child reproductive links and affected the rare nreproduction of human offspring. Since AIDS and its pathogens were recognized by scientists, people had realized its harm to human survival and development. Effective measures to prevent and treat AIDS had been formulated worldwide such as effective treatment drugs and prenatal care 
for pregnant women with HIV. So that pregnant women with HIV received continuous education and support throughout their pregnancy to achieve a healthy perinatal outcomes of women and newborns [1-4].

Midwifery students were a special group in the nursing specialty of medical specialties and the main force for future maternity work. Their work included nursing general pregnant women and pregnant women with HIV/AIDS. They not only need to prevent HIV/AIDS themselves, but also spread prevention knowledge abaut HIV/AIDS to the pregnant women. Therefore, they are not only the goals of AIDS health education, but they also bear inevitable responsibilities in the health education of AIDS prevention [3].

In order to effectively block the mother-to-child transmission of HIV/AIDS, midwives played a very important role in their delivery arrangements. Midwives must understand in who receives care and when, who provides care, the working conditions of those who provide care, coordination of care amongst different providers, where care is provided, the use of information and communication technology to deliver care, and quality and safety systems [4-6].

Midwives were the main force in future maternity work. They worked dealing with mothers and babies and perforemed the important task of preventing iatrogenic transmission every day. Their knowledge of preventing AIDS directly affected the prevention of AIDS and their own health $[3,7]$.

In order to understanding knowledge of HIV/AIDS mastered by the midwifery students, The investigation of AIDS-related knowledge was executed between 146 midwifery students who just entered the Zunyi Medical Collegel in 2014.

\section{Methods and Materials}

146 of midwife students enrolled in the 2014 grade of Zunyi Medical College were selected as the survey subjects. All of them were girls. The youngest was 18 years old and the oldest was 25 years old, with an average of $(20.23 \pm 2.53)$ years old.

The forms formulated with reference to relevant domestic and foreign materials of midwife students were issued uniformly by the teacher. They filled out the forms followed the requirements of the form items when students, they filled out the form, according to the requirements of the form items.

The forms filled out were anonymous. When they fill out the form they don't inspire, discuss, or communicate with each other. The items in the form were filled out independently by the students. The questionnaire was turned in on the spot when it was completed. There were 146 people in this survey, and 146 valid questionnaires were collected. The recovery rate was $100.0 \%$.

\section{Results}

Although 143/146 (97.94\%) midwife students knew that AIDS was an infectious disease, only1/146 (0.58\%) student can wrote HIV and AIDS word correctly. The definition of HIV was known to be $7 / 146$ (4.79\%). The abbreviation (AIDS) of acquired immunodeficiency syndrome was known to be 26/146 (17.80\%), 25/146 (17.12\%) Knew how AIDS was transmitted. $35 / 146(23.97 \%)$ Knew that the incidence of AIDS had increased in recent years in China. 12/146 (8.22\%) Knew that the AIDS cannot transmitted by mosquito biting. $10 / 146(6.85 \%)$ Knew that the AIDS cannot transmitted by swimming and squatting in the toilet. $15 / 146(10.2 \%)$ Knew that AIDS cannot be transmitted through shaking hands and eating together. 8/146 (5.4 8\%) Knew that the AIDS cannot transmitted by kissing. 86/146 (58.90\%) knew HIV can be transmitted to the fetus through the mother's placenta and infant through the mother's milk. $1 / 146(0.58 \%)$ Knew that women infected with HIV can get pregnant after intervention with anti-HIV drugs. (Table 1)

Table 1. SAwareness rate of AIDS knowledge among midwifery freshmen.

\begin{tabular}{lll}
\hline Knowledge area & Correct Answer No (\%) & Incorrect Answer No (\%) \\
\hline AIDS was an infectious disease & $143 / 146(97.94)$ & $3 / 146(2.06)$ \\
wrote HIV and AIDS word correctly & $1 / 146(0.58)$ & $145 / 146(99.42)$ \\
definition of HIV & $7 / 146(4.79)$ & $139 / 146(95.21)$ \\
Abbreviation of AIDS) & $26 / 146(17.80)$ & $120 / 146(82.20)$ \\
how AIDS was transmitted & $25 / 146(17.12)$ & $121 / 146(82.88)$ \\
Increasing AIDS incidenced in China & $35 / 146(23.97)$ & $111 / 146(76.03)$ \\
mosquito cannot transmitted AIDS & $12 / 146(8.22)$ & $134 / 146(91.78)$ \\
AIDS cannot transmitted by & & $136 / 146(93.15 \%)$ \\
Swimming and squatting toilet & $10 / 146(6.85 \%)$ & $131 / 146(89.80 \%)$ \\
AIDS cannot be transmitted through & & $138 / 146(94.52 \%)$ \\
shaking hands and eating together & $15 / 146(10.20 \%)$ & \\
AIDS cannot transmitted by kissin & $8 / 146(5.48 \%)$ & $60 / 146(41.10 \%)$ \\
AIDS can be transmitted by the & & \\
placenta and mother's milk & $86 / 146(58.90 \%)$ & \\
women with HIV may be pregnant & & \\
after intervention with anti-HIV drug 1/146 (0.58\%) & $145 / 146(99.42 \%)$ & \\
\hline
\end{tabular}




\section{Discussion}

Midwives were a special team of nurses whose job was to assist obstetricians and gynecologists to ensure the safety of mothers and newborns. They used their under- standing and knowledge of AIDS prevention and treatment to educate women in the perinatal care period and young women who want to become pregnant. Modern study of treatment showed that the married couples infected by HIV can delivered healthy babies throught effectively anti-AIDS drug blocking. It was a very important that midwives had a good grasp of HIV/AIDS prevention knowledge to prevent and treat HIV/AIDS during pregnancy and pregnancy. In order to decide whether to increase or decrease the learning content about HIV/AIDS in future medical courses, we did this survey before they studied medical courses.

The current incidence of AIDS in the China was around 9\%. According to statistics, there were about 1.25 million people with HIV in the China that there were 1.4 billion people in the country. The main way of transmission of AIDS was through extramarital sex without protective measures. The main way of transmission of AIDS for men was male homosexuality. Other transmission ways were invasive operations in informal institutions, intravenous drug use, and mother-to-child transmission. AIDS cannot be spread through the sweat, saliva, tears, and urine.

In a hospita, research by Liu Zhimei [8] find out that there wrer 868 cases of HIV positive antibody patients through HIV screening in 54784 cases inpatients of internal medicine requiring blood transfusion and 271,431 cases of surgical inpatients requiring surgery and 49,697 cases of Sexually transmitted diseases (STD) outpatients in STD clinics from January 2002 to December 2012. Before admission all 375912 cases did not provide to the doctor with an HIV-positive medical history. Among 868 cases of HIV positive antibody patients, there were 7 cases of college students and 3 cases of middle school student. It indicated that the epidemic situation of HIV infection is still severe due to insufficient sex knowledge education and awareness of the harmfulness of HIV infection. College students and middle school students are at greater risk of HIV infection. College students had been listed as the high-risk group of HIV/AIDS by National Health Commission and of people's Republic of China [9-11].

The awareness rate of/HIVAIDS among new midwives was worrying according to the survey results, Although the vast majority of students (97.94\%) knew AIDS was an infectious disease, but they know a little about the detailed knowledge of HIV/AIDS. For example, no student could write the full English name of HIV/AIDS correctly, only 2 students could answered the three transmissive routes of AIDS completely, and only 25 students $(17.12 \%)$ could answer the correct one or two routes. There had been poor understanding whether HIV can spread by the mosquito bites, the swimming pools, shaking hands, eating, kissing and toilets.

During the 12 years of primary and secondary education before entering university, Chinese college students spended a lot of time on learning all kinds of examinative knowledge. Education of sexual knowledge was not well carried out in many schools. Boys and girls have less knowledge about sex, especially about infectious diseases that can be transmitted through sexual contact such as HIV/AIDS. A little of the sexual knowledge was learned only occasionally from the press, the news and the television. The midwife profession was a special group in the nursing profession. They were the target of AIDS health education before working and instructor of HIV/AIDS prevention after working with pregnant women and newborns every day, It's of great significance to improve the quality of HIV/AIDS prevention and treatment in China if midwife had mastered the knowledge of HIV/AIDS prevention and control and carry out publicity among pregnant women especially in preventing mother-to-child transmission of HIV/AIDS.

A survey had been conducted on the knowledge of HIV/AIDS among university students in some domestic colleges and universities in the China. The results were not satisfactory. The knowledge of prevention and treatment of HIV/AIDS was related to sexual knowledge education and HIV/AIDS knowledge rather than with the increasing of learning years [12-15]. Our investigatiive results shew that it was very important to strengthen the HIV/AIDS knowledge education of midwives. We will invite relevant experts to carry out prevention and treatment lectures of HIV/AIDS to improve the ability of midwives to prevent and treat HIV/AIDS.

Midwives and obstetricians played a very important role in interrupting the transmission of HIV/AIDS. Prevention and treatment courses of HIV/AIDS should be studyed by the midwives during their medical school and Strengthened in the work. These midwives then organized pregnant women and parturients to learn about the knowledge to prevented HIV/AIDS. These are very important to prevent the mother-to-child transmission of HIV/AIDS and the mother's physical and mental health [16, 17]. The HIV/AIDS prevention and treatment courses are recommended in the midwife profession.

\section{Conflict of Interests}

The authors declare that there is no conflict of interests regarding the publication of this paper.

\section{Acknowledgements}

I give a great appreciation and opportunity to thank this study supported by stadholder funding of Guizhou Province (funding number: 040-109) and Zunyi Midical College for initiating this research program and financial support (Zunyi Midical College [2017] No: 005). The deep thanks go to department of nursing of Zunyi Midical Collegeh sciences community and research coordination office for spent their valuable time during enquiry of this study. Wish to express my sincere thanks to all people who were involved interview, and those responded to the questionnaires for devotion of their 
time and energy. The last but not the least I am thankful to Zhang Xinjiang professor and my son for their moral support and patience.

\section{References}

[1] Jonell EP, Lunthita MD, Nelly DM, et al. Implementing centering pregnancy group prenatal care for minority women living with HIV at an Urban University Hospital [J] Journal Midwifery Womens Health; 2019 Jul; 64 (4): 451-461.

[2] Michael SS, Rajesh TG, Jennifer FH, et al. Antiretroviral drugs for trestment and prevention of HIV infection in adults: 2020 rocommendations of the Interational Antivial Panel [J] JAMA. 2020 Oct 27; 324 (16): 1651-1669.

[3] Gideon L P, Vera C, Jean D C, et, al. Sustaining hope: A narrative inquiry into the experiences of hope for nurses who work alongside people living with HIV in Ghana [J]. Nurs Open. 2020 Mar 10; 7 (3): 879-886.

[4] Bipasha C, Maria S, Dolores F J; et, al. Pregnancy among HIV-serodiscordant couples: case report of vertical transmission and retrospective case series Curr [J]. HIV Res. 2020 Dec 31. doi: 10.2174/1570162X18999201231211658. Online ahead of print.

[5] Claudia B M, Hannah B, James B; et al. Nurses in expanded roles to strengthen community-based health promotion and chronic care: policy implications from an international perspective; A commentary Isr J Health Policy [J]. Res 2018 Oct 12; 7 (1): 64. doi: 10.1186/s13584-018-0257-5.

[6] Fatemeh O, Farzaneh K, Forough R; et al. Qualitative study of HIV related stigma and discrimination: What women say in Iran [J]. Electron Physician, 2017 Jul 25; 9 (7): 4718-4724.

[7] Vincent J T, Rhoderick M, Michelle M G; et al. 24-month HIV-free survival among HIV-exposed Infants in Lesotho: the PEAWIL cohort study. J Int AIDS Soc 2020 Dec; 23 (12): e25648. doi: 10.1002/jia2.25648.

[8] Liu ZM, Wu H, Luo XH, et al. HIV antibody screening in a hospital from 2002 to 2014 [J], Journal Clinical Dermatology October, 2016, 45 (10); 698-700.

[9] Agustín C, Simon L, Cristian AH, et al. Delivery arrangements for health systems in low-income countries: an overview of Systematic review Cochrane Database Syst Rev. 2017 Sep 13; 9 (9): CD011083.

[10] Lewis JE, M alow RM, Irel and SJ. HIV/AIDS Risk in heterosexualcollege st-udents-A review of a decade of literature [J]. J Am CollHealth, 1997, 45 (4): 147-158.

[11] Lal SS, Vasan RS, Sanna PS, et al. Knowledge and attitude of col-lege students in Kerala towards HIV/AIDS, sexually transmitteddiseases and sexuality [J]. Natl Med J Inda, 2000, 13 (5); 231-236.

[12] Yun L, Jia H. Status of HIV/AIDS knowledge cognition amoung medical school students [J]. China Medical and Pharmacy, 2011, (18); 26-28.

[13] $\mathrm{Lu} \mathrm{B}, \mathrm{Lu} \mathrm{HG}$, Liu ZG,. Investigation and analysis of prevention knowledge of HIV/AIDS among college students in Guiyang [J]. Journal of Guiyang Medical College. 2006, 31 (6); 552-553.

[14] Xu JS, Zhang DL, Chen H. Survey and analysis of HIV/AIDS knowledge and attitudes among junior college students in Shanghai [J]. Reproduction \& contraception; 2006, 26 (10); 631-633.

[15] Hou QY, Liu D, Long J, Asurvey of the cognition of AIDS and attitudes of the people infected with HIV among studing college student [J]. Guangzhou medicine, 2015, 46 (3); 36-39.

[16] Brandon A K, Rimel N M, Linda M; et al. Exploring patterns and predictors of suicidal ideation among pregnant and postpartum women living with HIV in Kilimanjaro, Tanzania [J]. AIDS. 2020 Sep 1; 34 (11): 1657-1664.

[17] Winnie M, Cephas M, Obey S; et al. Prevention of mother-to-child transmission activities after one-off clinical mentorship training in selected health facilities, Zimbabwe: 2014-2018 [J]. Pan Afr Med J. 2020 Jul 1; 36: 146. doi: 10.11604/pamj.2020.36.146.19542.eCollection 2020. 